\title{
Impact of COVID-19 on postgraduate surgical training: the trainees' perspective
}

Pradeep K Wijerathne ${ }^{1,6^{*}}$, Kushan Nanayakkara, ${ }^{2,6^{*}}$, Oshan Basnayake ${ }^{3,6}$, Nuwan Gunapala ${ }^{4,6}$, Umesh Jayarajah ${ }^{5,6}$ 'Department of Surgery, National Hospital of Sri Lanka, Colombo, Sri Lanka

${ }^{2}$ Department of Upper Gastrointestinal Surgery, Royal Derby Hospital, Derby, UK

${ }^{3}$ Department of Oncosurgery, Apeksha Hospital, Maharagama, Sri Lanka

${ }^{4}$ Department of Breast Surgery, Newham University Hospital, Barts Health, London, UK

${ }^{5}$ Department of Urology, Colombo South Teaching Hospital, Kalubowila, Sri Lanka

${ }^{6}$ Postgraduate Institute of Medicine, University of Colombo, Sri Lanka

*Equal contribution

Keywords: COVID-19; SARS-CoV-2; postgraduate surgical training; trainees' perspective

\section{Introduction}

COVID-19 was declared a global pandemic by the World Health Organization on 11th March 2020 [1]. As of 8th of December 2020, more than 60 million cases and 1.4 million deaths have been reported globally. In Sri Lanka, more than 28,000 cases and 140 deaths have been registered so far [2]. Moreover, Sri Lanka is currently facing a second major outbreak. COVID-19 pandemic has caused profound effects on almost all the aspects of modern human life, inducing the global economy and the livelihood of the general public $[3,4]$. A significant impact on all aspects of health care including the surgical specialities has been observed [5]. With the ongoing global pandemic and lack of data on long term effects of vaccines, the future direction of the pandemic remains uncertain [6]. We aim to provide the trainees' perspective on the impact on postgraduate surgical training during the pandemic at local and overseas settings.

\section{Impact on local surgical training}

The impact on medical training has been observed in both undergraduate and postgraduate training programs. The initial outbreak of COVID-19 in Sri Lanka negatively affected postgraduate surgical training for over two months before the resumption of near-normal routine surgical services. The impact due to the current second outbreak may be more severe due to the wider spread within the community, possibly exceeding the chances of containment.

In surgical specialities, hands-on experience to develop technical skills is a crucial component among other aspects of training. Changes in the healthcare system were made to minimize potential exposure and spread among patients and staff members and to utilize the limited resources productively. This approach also avoided major disruptions of emergency surgical services. Limiting care to urgent cases resulted in a smaller number of surgical patients being

Correspondence: Umesh Jayarajah

E-mail: umeshe.jaya@gmail.com

(iD https://orcid.org/0000-0002-0398-5197

Received: 23-11-2020 Accepted: 16-12-2020

DOI: http://doi.org/10.4038/sljs.v38i3.8769 assessed and operated by trainees. Patients awaiting elective surgeries wished to postpone the operation. An analysis of a number of routine cases performed during the first 11 months in 2019 and 2020 at both surgical units in District General Hospital Kegalle showed a reduction from 1574 to 1165 cases [a $25 \%$ reduction]. Some patients tend to default follow up due to the fear of exposure to the virus. Higher risk of exposure and transmission among surgeons and trainees when performing various types of surgeries and endoscopic procedures also led to limitations in laparoscopy and endoscopy procedures. In an international survey among trainees across 63 countries, approximately 94\% experienced lesser case volume during the pandemic. The greatest reduction was seen for colonoscopy procedures [7]. Therefore, trainees faced limited exposure and hands-on training in these aspects. Lectures, conferences, other educational activities were also limited to minimize transmission. Trainees were also facing difficulty in conducting clinical research due to the reduced patient load and risk of exposure to the virus. Depending on the prevalence of the disease, there were regional variations of the above mentioned factors. For example, an analysis of a number of admissions during the first 10 months in 2019 and 2020 in a surgical ward in District General Hospital Chilaw showed a reduction from 6081 to 3868 cases [a 36\% reduction], whereas, in Base Hospital Horana, a reduction from 3969 to 3511 [an $11.5 \%$ reduction] was seen.

The impact on training also depends on the stage of surgical training and the subspecialty. The first year of the pre-MD training program mainly focuses on acquiring basic surgical skills and knowledge of fundamental surgical concepts. Clinical rotation with shorter durations has been affected more than those with relatively longer durations where there is time for some degree of compensation. For example, trainees doing short appointment of one or two months during the lockdown with theatre restrictions were exposed to a fewer number of cases. In a tertiary care urology unit at Colombo South Teaching Hospital, the average number of cases per month reduced from 64 to 30 [greater than 50\% reduction] during theatre restrictions. The trainees awaiting foreign training after completion of local training are also affected. The risk of travelling to disease prevalent countries,

The Sri Lanka Journal of Surgery 2020; 38(3): 53-55 
delay in processing the requirements and completion of training are some of the negative consequences. The MD Surgery examinations have been standardized and well recognized by the international surgical community. Due to the current travel restrictions, difficulties have been encountered in inviting foreign examiners to maintain the standardization procedures.

\section{Challenges faced by the overseas trainees in the UK}

Overseas training is often challenging for many surgical trainees due to the differences in the patients, trainers, setting and completely different living environment. In addition, the effects of the ongoing pandemic caused unexpected greater challenges for the trainees.

National Health Service [NHS] in the UK is a highly organized functional unit which spends billions of pounds annually to cater the public health needs. Despite the existing strategies and resources, most departments struggled to continue their routine activities when hospitals were flooded with COVID patients during the pandemic. Except in highly specialized surgical units in a limited number of hospitals, all others units were forced to make a difficult decision to cancel or postpone their elective surgical activities including theatres, clinics and multidisciplinary team meetings. Although emergencies could carry on, most patients were managed conservatively with minimal operative input. In a global online survey on the treatment of acute appendicitis, conservative measures for uncomplicated and complicated appendicitis were utilised by 2.4 and $6.6 \%$ of surgeons respectively before, but 5.3 and $23.7 \%$, during the pandemic [8]. The main goal of overseas training is to broaden the horizons of existing knowledge with new experiences and techniques in the field of interest. However, this was severely affected during the COVID pandemic. Most trainees were redeployed as ward doctors or to a speciality of need, hampering the surgical training and acquisition of surgical skills. Some were deployed to the front-line of managing non-surgical COVID patients without proper training, and initially, without adequate personal protective equipment.

The safety of the family was a major concern. Most trainees living with their family in the UK lived in fear of exposing their family to the virus when returning home after working in a high-risk environment. This included the more vulnerable elderly parents and relatives who were forced to stay in the UK until the travel restrictions were sorted. Due to the travel restrictions, reaching the family in Sri Lanka was not possible in case of an emergency. Living in an unfamiliar environment with restricted travelling and leisure activities during lockdown resulted in increased levels of psychological stress, especially among the children.
It is likely that COVID-19 will affect the future overseas trainees as well. Most of the trusts may extend the contracts of existing local employees, which often closes doorways to overseas trainees. As most hospitals are facing financial difficulties, the recruitment of new overseas trainees may become limited.

\section{Positive aspects amidst the COVID-19 pandemic}

Every dark cloud has a silver lining, and similarly, COVID 19 has taught some important lessons that cannot be ignored. Irrespective of the initial struggle, we witnessed the adaptive capability of the health sector in general, both in Sri Lanka and overseas.

The trainees received experience working in a resourcelimited setting with reduced staff. With the above limitations, prioritization of theatre lists and other surgical care was another aspect of learning and experience. With the limited number of the caseload, trainees received more supervision during surgery while operating with the consultants than engaging in clearing out heavy theatre lists. Operating with personal protective equipment was also a challenging new experience with the limitation in ergonomics and visual perception. Due to a reduction in clinical work and responsibilities, trainees had more time for writing, research, self-study, leisure time activities and family commitments.

The efforts to mitigate the negative effects in surgical training were carried out by the College of Surgeons, Postgraduate Institute of Medicine and various other clinical societies locally and internationally. Web-based video conferencing to facilitate educational activities were carried out in different forms like case-based discussions and topic discussion. The annual academic sessions were held virtually which gave a different experience to the surgical community. Many academic societies granted free access to their resources and learning materials to improve medical education worldwide. Improved availability and free access to operative video libraries and the development of comprehensive online learning programs by leading surgical colleges and institutions were welcome changes. Advantages of web-based activities also include cost-effectiveness of conducting activities for the organizers and also for the participants.

Although the usual clinical researches have been affected by the pandemic, web-based global platforms have given opportunities for the surgeons and surgical trainees to engage in collaborative researches $[9,10]$. During this pandemic, the COVIDSurg Collaborative conducted evidence-generating global studies with the collaboration of surgeons and surgical trainees globally using a web-based platform $[11,12]$.

\section{Conclusion}


Surgical speciality is always full of challenges. However, time to time we do face some exceptional challenges such as the current COVID-19 pandemic. The ongoing outbreak may hinder the usual training process and the global pandemic may continue onto the foreseeable future. Embracing the positive aspects and implementing alternative options to mitigate the negative effects on surgical training would be the way forward.

All authors disclose no conflict of interest. The study was conducted in accordance with the ethical standards of the relevant institutional or national ethics committee and the Helsinki Declaration of 1975, as revised in 2000 .

\section{References}

1. Borges do Nascimento IJ, Cacic N, Abdulazeem HM, von Groote TC, Jayarajah U, Weerasekara I, et al. Novel coronavirus infection [COVID-19] in humans: a scoping review and metaanalysis. Journal of Clinical Medicine. 2020;9[4]:941. https://doi.org/10.3390/jcm9040941

2. World Health Organization. Coronavirus disease [COVID-2019] situation reports. 2020.

https://www.who.int/emergencies/diseases/novel-coronavirus2019/situation-reports

3. Borges do Nascimento IJ, von Groote TC, O'Mathúna DP, Abdulazeem HM, Henderson C, Jayarajah U, et al. Clinical, laboratory and radiological characteristics and outcomes of novel coronavirus [SARS-CoV-2] infection in humans: A systematic review and series of meta-analyses. PloS One. 2020;15[9]:e0239235. https://doi.org/10.1371/journal.pone.0239235

4. Jayasinghe R, Ranasinghe S, Jayarajah U, Seneviratne S. Quality of online information for the general public on COVID-19. Patient Education and Counseling. 2020;103[12]:2594-7. https://doi/10.1016/j.pec.2020.08.001
5. Jayasinghe R, Jayarajah U, Seneviratne S. Consensus on perioperative surgical practice during the COVID-19 pandemic: an appraisal of the literature. Sri Lanka Journal of Surgery. 2020;38[2]:57-61.http://doi.org/10.4038/sljs.v38i2.8738

6.Seneviratne SL, Jayarajah U, Abeysuriya V, Rahman A, Wanigasuriya K. COVID-19 Vaccine Landscape. Journal of the Ceylon College of Physicians. 2020;51:120-131

7. Pawlak KM, Kral J, Khan R, Amin S, Bilal M, Lui RN, et al. Impact of COVID-19 on endoscopy trainees: an international survey. Gastrointestinal Endoscopy. 2020;92[4]:925-35. doi: 10.1016/j.gie.2020.06.010

8. Ielpo B, Podda M, Pellino G, Pata F, Caruso R, Gravante G, et al. Global attitudes in the management of acute appendicitis during COVID-19 pandemic: ACIE Appy Study. The British Journal of Surgery. 2020: In press. https://doi.org/10.1002/bjs.11999

9. Collaborative C. Preoperative nasopharyngeal swab testing and postoperative pulmonary complications in patients undergoing elective surgery during the SARS-CoV-2 pandemic. British Journal of Surgery 2020: In press. https://doi.org/10.1093/bjs/znaa051

10.Glasbey JC, Bhangu A, Collaborative C. Elective cancer surgery in COVID-19-free surgical pathways during the SARS-CoV-2 pandemic: an international, multicenter, comparative cohort study. Journal of Clinical Oncology. 2020:JCO. 20.01933. doi: $10.1200 / \mathrm{JCO} .20 .01933$

11.Collaborative C. Delaying surgery for patients with a previous SARS-CoV-2 infection. The British journal of surgery. 2020;107:e601-e2. https://doi.org/10.1002/bjs.12050

12.Collaborative C. Outcomes from elective colorectal cancer surgery during the SARS-CoV-2 pandemic. Colorectal Disease. 2020: In press. https://doi.org/10.1002/bjs. 12050 